Pia Gabriel-Schärer / Marion Baud-Lavigne

\title{
Prix SEVAL 2021: Würdigung der Nominierten und Laudatio für den Preisträger
}

Beitragsart: Mitteilungen

Zitiervorschlag: Pia Gabriel-Schärer / Marion Baud-Lavigne, Prix SEVAL 2021: Würdigung der Nominierten und Laudatio für den Preisträger, in: LeGes 32 (2021) 3 
[1] Die Jury des Prix SEVAL hatte in diesem Jahr die schöne und anspruchsvolle Aufgabe, zwischen drei sehr guten Arbeiten zu entscheiden, wem der Prix SEVAL 2021 überreicht werden soll. Alle drei Arbeiten haben den Kriterien weitgehend entsprochen und überzeugten hinsichtlich Wissenschaftlichkeit, Praxisrelevanz, Innovation und Originalität sowie bei der Form der Aufbereitung und verdienen in den folgenden Abschnitten eine Würdigung.

[2] Dr. Regina Jensen hat ihre Abschlussarbeit des CAS in Evaluation an der Universität Bern 2020 zum Thema «Die Perspektive von Evaluierenden und Auftraggebenden auf Empfehlungen in Evaluationsberichten» eingereicht. Sie bearbeitet darin die relevante Frage, wie Empfehlungen gestaltet werden können, damit sie akzeptiert und umgesetzt werden. Regina Jensen bearbeitet diese Frage sowohl theoretisch als auch empirisch. Sie liess die Merkmale von Empfehlungen in zwei Befragungen - eine bei Auftraggebenden von Gesundheitsförderung Schweiz und eine bei Evaluierenden - bewerten. Resultat war eine empirische Beurteilung der Merkmale guter Empfehlungen, was sowohl für Auftraggebende wie auch für Evaluierende für die praktische Arbeit von grosser Relevanz ist.

[3] Auch bei der zweiten nominierten Arbeit handelt es sich um eine Abschlussarbeit des CAS in Evaluation an der Universität Bern 2020. Marina HäUsermann hat sich damit beschäftigt, wie sich die aktuelle Covid-19-Pandemie auf die Evaluationstätigkeit auswirkt - ein Thema, das noch wenig diskutiert wurde. Die Arbeit trägt den Titel «Development of Guidelines on «Evaluation in Times of Covid-19:: Based on the analysis of recent publications and to date recommendations by the international evaluation community». Marina HäUSERMANn hat eine Literaturrecherche mit anderen originalen Quellen wie Blogs und Webinaren kombiniert. Sie hat die Erkenntnisse mit der Evaluationsgemeinschaft diskutiert und in einer handlichen Checkliste verdichtet, welche zukünftig für die Reflexion der Planung einer Evaluation eingesetzt werden kann.

[4] Der Prix SEVAL 2021 wurde schlussendlich an Michael Debétaz für seine Arbeit «Big data, machine learning et évaluation: la data science au service des administrations» übergeben ebenfalls eine Abschlussarbeit des CAS in Evaluation ${ }^{1}$. Die Jury möchte damit ein Zeichen setzen. Heutzutage werden immer mehr Evaluationen durchgeführt, diese sind jedoch oft standardisiert und verwenden altbewährte Untersuchungsinstrumente. Michael Debétaz' Arbeit setzt an diesem Punkt an und erinnert uns an eine der wichtigsten Tugenden der Evaluation: Evaluation ist in erster Linie Methodik, eine Technik, um einen Gegenstand zu durchleuchten. Der Autor stellt sich aus diesem Grund die Fragen, was die digitale Revolution für die Evaluationstätigkeit bedeutet und welchen Beitrag sie bei der Durchführung von Evaluationen leisten kann. Die Arbeit besticht nicht nur darin, dass sie Schlagwörter wie Big Data und Machine Learning verständlich erklärt, sondern auch, dass sie ihnen einen Sinn in der Evaluationswelt geben kann. Michael Debétaz gibt dabei einen wichtigen Einblick in die potentielle Verwendung von fortgeschrittenen Methoden wie Machine Learning für Evaluatoren und Evaluatorinnen. Anhand von konkreten Beispielen erläutert der Autor wie quantitative Methoden als Evaluationsinstrumente genutzt werden können, um öffentliche Massnahmen zu erfassen und zu evaluieren. Die Beispiele sind sehr gut illustriert und enthalten sogar die verwendeten Codes, die jede Evaluatorin und jeder Evaluator für sich verwenden kann. Wie es nicht anders sein könnte, ist die Arbeit nicht auf Papier verfasst worden, sondern wird per Open Access im Internet zur Verfügung gestellt ${ }^{2}$. Ins-

\footnotetext{
https://www.seval.ch/publikationen-prix-seval/prix-seval/

https://greval.ch/big-data-machine-learning-et-evaluation-1-introduction/
} 
gesamt würdigt die Jury des Prix SEVAL die Arbeit als sehr wissenschaftlich, innovativ und mit hoher Praxisrelevanz für die Evaluationsgemeinschaft. Es wäre wünschenswert, wenn damit ein Dialog über die kritische Einordnung und Würdigung der Nutzung von Big Data und Machine Learning in unserer heutigen Zeit lanciert werden könnte.

\section{Ausschreibung des Prix SEVAL 2022}

[5] Um die wissenschaftliche Auseinandersetzung mit Evaluation zu fördern hat die SEVAL den Prix SEVAL geschaffen. Mit diesem Preis werden einmal jährlich wissenschaftliche Arbeiten ausgezeichnet, die einen hervorragenden Beitrag zur Weiterentwicklung von Theorie und Praxis der Evaluation oder zur Verwendung von Evaluation liefern.

[6] Der Prix SEVAL wird jeweils anlässlich des SEVAL-Jahreskongresses verliehen. Der Preis ist mit CHF 3000.- dotiert und beinhaltet auch die Veröffentlichung eines Beitrages in LeGes.

[7] Teilnahmeberechtigt sind Qualifikationsarbeiten aller Stufen, in Fachzeitschriften publizierte Artikel, Fachbücher oder Evaluationsstudien von Studierenden, Forschenden und anderen wissenschaftlich tätigen Personen in der Schweiz, die einen Bezug zur Evaluation aufweisen. Arbeiten können ab sofort beim SEVAL-Sekretariat eingereicht werden (secretariat@seval.ch), Einsendeschluss ist der 2. April 2022. Sie können auf Deutsch, Französisch, Italienisch oder Englisch verfasst sein. Die eingereichten Arbeiten werden auf ihre Wissenschaftlichkeit, Originalität, Praxisrelevanz und Form beurteilt.

[8] Die Jury für den Prix SEVAL setzt sich wie folgt zusammen:

- Prof. Pia Gabriel-Schärer, Hochschule Luzern, Soziale Arbeit, Luzern (Präsidentin der Jury)

- Prof. Pirmin Bundi, IDHEAP Universität Lausanne

- Marion Baud-Lavigne, Evaluatorin und Projektleiterin, Parlamentarische Verwaltungskontrolle

- Nicole Kaiser, Senior Projektleiterin, econcept AG

[9] Die offizielle Ausschreibung, das detaillierte Reglement, sowie weitere Informationen zum Prix SEVAL sind auf der SEVAL-Webseite (https://www.seval.ch/publikationen-prix-seval/prixseval/) aufgeschaltet.

Prof. Pia Gabriel-Schärer, Psychologin und Evaluatorin, Hochschule Luzern - Soziale Arbeit, Luzern.

Marion Baud-Lavigne, Evaluatorin und Projektleiterin, Parlamentarische Verwaltungskontrolle, Bern.

Ce texte a été rédigé en se basant sur des commentaires écrits également par Nicole Kaiser et Pirmin Bundi. 